\title{
Uncommon course of the ulnar artery through the pronator teres muscle
}

\author{
Katerina Vymazalová, ${ }^{2}$ Lenka Vargová,, ${ }^{1}$ Marek Joukal' \\ 'Division of Medical Anthropology, Department of Anatomy, Faculty of Medicine, Masaryk University Brno, \\ Czech Republic.
}

\section{ABSTRACT}

In this paper, we describe a very rare variant in the course of the ulnar artery that we encountered in dissecting the right upper limb of a 74-year-old man. The ulnar artery arose standardly from the brachial artery in the cubital fossa. However, its ensuing course differed from the norm. The artery entered together with the ulnar vein and median nerve into the pronator canal (between the humeral and ulnar heads of the pronator teres). Further, the ulnar artery descended classically to the ulnar side of the forearm between the flexor carpi ulnaris and flexor digitorum superficialis. Knowledge of this variation in the course of the ulnar artery may have significance in clinical practice because accumulation of anatomical structures in the pronator canal could be a predisposing factor for the compression of nerve or blood vessels.

Keywords: anatomical variation; median nerve; pronator canal; pronator teres muscle; ulnar artery.

\section{INTRODUCTION}

The ulnar artery is one of the two terminal branches of the brachial artery. In most cases, it begins in the cubital fossa, then passes under the group of the superficial flexors of the forearm (including the pronator teres) and continues distally between the flexor carpi ulnaris and the flexor digitorum superficialis together with the ulnar nerve. The ulnar artery supplies the frontal and dorsal group of muscles of the forearm and skin in the ulnar half of the forearm. ${ }^{1}$

Variable courses of the ulnar artery have been reported by a number of authors, for example by RodríguezNiedenführ ${ }^{2}$ or Kumar et al. ${ }^{3}$ This paper describes one of the very rare variations in the course of this artery.

\section{CASE REPORT}

A case of an uncommon course of the ulnar blood vessels was observed during anatomical dissection of the right upper limb of a 74-year-old man. The cadaver had been fixed with the following fixative: $96 \%$ ethyl alcohol, $100 \%$ glycerine, 35-40\% formalin, 5\% phenol. The anatomic structures were dissected on this limb by conventional autopsy procedures used in the teaching of medical students at the Department of Anatomy, Medical Faculty, Masaryk University Brno. ${ }^{4}$

On the studied specimen the pronator teres originated standardly by two heads - the humeral (from the medial epicondyle of the humerus) and the ulnar (from the coronoid process of the ulna). Also, the median nerve ran as usual between the two heads of the muscle. Usually, the ulnar artery should arise from the brachial artery in the cubital fossa, where it is in contact with the median nerve in the range of about $2.5 \mathrm{~cm}$. Distally the nerve crosses the artery and reaches the pronator canal while the artery should pass under the group of

Correspondence: Katerina Vymazalová, Department of Anatomy, Faculty of Medicine, Masaryk University Brno, Czech Republic. Email: vargova@med.muni.cz. 
the flexors of the forearm, including the pronator teres. The ulnar artery and median nerve are separated in the further course by the ulnar head of the pronator teres. ${ }^{1}$ In our case, the artery together with the ulnar vein and the median nerve entered the pronator canal, i.e. the gap between the two heads of the pronator teres (Figure 1). Beneath the pronator teres there arose from the ulnar artery two branches, more proximally the recurrent ulnar artery toward the medial epicondyle of the humerus and more distally the common interosseous artery leading to the antebrachial interosseous membrane. The following course of the ulnar artery between the flexor ulnaris and flexor digitorum superficialis toward the hands matched the norm. The radial artery exhibited a standard course: after originating from the brachial artery, it continued distally in the groove between the brachioradialis and pronator teres and its dilatation was not observed there. No other variations were observed in the upper extremity.

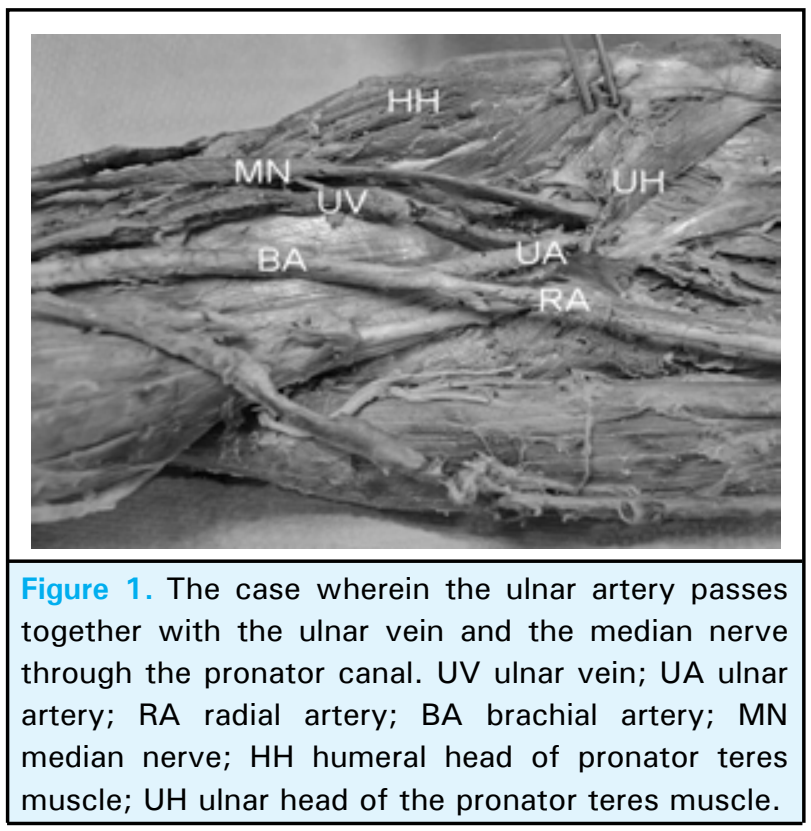

\section{DISCUSSION}

The pronator canal is one of the clinically most important anatomical structures of the upper extremity. It is a gap between the humeral and the ulnar heads of the pronator teres through which passes the median nerve in $80-95 \%$ of cases, as stated by Mori ${ }^{5}$ or Lee and LaStayo. ${ }^{6}$ This space is commonly the point where the entrapment syndrome of the median nerve known as pronator teres syndrome, which occurs in about $9.2 \%$ of cases, arises. ${ }^{6}$ Compression of the median nerve between the heads of the pronator teres occurs primarily in muscle hypertrophy, which can appear mainly among people engaged in repeated pronation with flexion of the fingers. Less frequent causes include abnormalities in the development of tendons or muscles, as well as changes caused by trauma of the surrounding structures, such as post-traumatic hematoma and fracture. ${ }^{7}$

The common course of the ulnar blood vessels and the median nerve in the pronator canal may in certain circumstances be a predisposing factor for compression not only of the nerve but also of the blood vessels. The probability of entrapment syndrome in this area is increased by the fact that more structures than usual pass through this canal.

Robertson and Neumeister ${ }^{8}$ had described an example of median nerve compression caused by an anomalous ulnar artery, but that was in the distal part of the forearm. A common course for the ulnar artery and vein and the median nerve through the pronator canal cannot be considered as a frequent variant, because it is not commonly reported in the professional literature. The more common variants described in the literature include a high origin of the ulnar artery from the brachial artery or presence of the superficial ulnar artery. Sometimes, the ulnar artery may also be absent or duplicated ${ }^{2}$. One of the important deviations of the branching pattern of the ulnar artery is persistent median artery, which is a transitory artery that occurs in embryonic development and accompanies the median nerve. This artery usually arises from the common interosseous artery the anterior interosseous artery, or directly from the ulnar artery. It joins the median nerve after its passage through the pronator canal and in some cases accompanies the nerve through the carpal canal to the palm. ${ }^{9}$ In our case, the ulnar artery accompanied the median nerve only through the pronator canal, and it was separated through the following course of the nerve. It is therefore clear that the variant we observed is not identical with the median artery.

In the studied case, only the death certificate was available and thus it was not possible on the basis of medical records to confirm any oppression of the median nerve or of the ulnar artery. However, cases of compression of arteries between the muscle fibers, followed by upper limb ischemia and claudication are not exceptional in clinical practice. ${ }^{10}$ Upper extremity ischemia is less common than is that in the lower limbs (the two occur in a ratio of about 1:9), and, when it occurs, it most often relates to the area which is supplied by the subclavian artery. Forearm arteries are rarely affected by ischemia. ${ }^{11}$ Sometimes, oppression of the artery and nerve can occur together. Compression of the brachial artery and the median nerve has been described, for example, by George and Nayak. ${ }^{12}$

Kumar et al. ${ }^{3}$ have mentioned a possibility for entrapment 
syndrome of the ulnar artery along its course through the pronator teres. The artery they described, in contrast to our case, had not passed between the two heads of the muscle, but rather beneath a third, accessory head. A case similar to ours was described by Koshy et al. ${ }^{13}$ In that instance, the brachial artery had passed together with the median nerve between two heads of a tripleheaded pronator teres and beyond the pronator teres the brachial artery had then divided into other branches.

Clinical manifestations of arterial compression may have been similar in the two cases, as blood flow in the ulnar artery would have been affected by contraction of the pronator teres.

During the human ontogenesis, the ulnar artery derives from the primitive axial artery. Rodríguez-Niedenführ et al. ${ }^{14}$ states that the arterial pattern of the upper limb develops from an initial capillary plexus due to the maintenance, enlargement and differentiation of certain capillary vessels, and the regression of others. Arterial variations may be explained on the basis of this theory by modifications of the normal pattern of capillary maintenance and regression. It is obvious, that chemical factors such as oxygenation and nutrient requirements, as well as haemodynamic forces, and naturally genetic mechanism can also determinate the arterial pattern.

From a clinical point of view, the course of the ulnar artery through the pronator canal is important. This anatomical arrangement may increase the risk for compression of the ulnar artery, as well as of the median nerve, and it is necessary to take this possibility into account when making the differential diagnosis of upper limb ischemia and stenotic syndromes of the median nerve.

\section{REFERENCES}

1. Drake RL, Vogl W, Mitchell AWM, Gray H, Gray H. Gray's anatomy for students, Churchill Livingstone/Elsevier, Philadelphia, PA. 2010

2. Rodríguez-Niedenführ M, Vazquez T, Nearn L, Ferreira B,

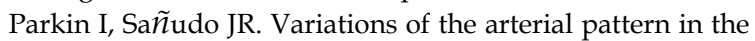
upper limb revisited: a morphological and statistical study, with a review of the literature. J Anat. 2001;199(5):547-566.

3. Kumar N, Rao SS, Aithal A, Nayak SB. Possible entrapment of the ulnar artery by the third head of pronator teres muscle. Rev Arg Anat Clin. 2012;4(3):102-105.

4. Žlábek K, Páč L. Pitevní cvičení z normální anatomie. Masarykova univerzita, Brno. 2011 (in Czech)

5. Mori M. Statistics on the Musculature of the Japanese. Okajimas Folia Anat Jpn. 1964; 40:195-300.

6. Lee MJ, LaStayo PC. Pronator syndrome and other nerve compressions that mimic carpal tunnel syndrome. J Orthop Sports Phys Ther. 2004;34(10):601-609.

7. Andreisek G, Crook DW, Burg D, Marincek B, Weishaupt D. Peripheral neuropathies of the median, radial, and ulnar nerves: MR imaging features. Radiogr Rev Publ Radiol Soc N Am Inc. 2006;26(5):1267-1287.
8. Robertson GA, Neumaister MW. Ulnar artery entrapment of the median nerve. Can J Plast Surg. 1999;7(1):35-37.

9. Rodríguez-Niedenführ M, Sañudo JR, Vázquez T, Nearn L, Logan B, Parkin I. Median artery revisited. J Anat. 1999;195(1):57-63.

10. Mahato NK. Entrapment of the Median Nerves and Brachial Arteries in the Lower Arms Bilaterally and Additional Origin of Biceps Brachii Muscle: Case Report. Int J Morphol. 2010;28(4):1241-1244

11. Staněk F. Ischemická choroba horních končetin, Angiologie pro praxi, Karetová D, Staněk F (Eds), Maxdorf, Praha. 2007;pp 109-112. (in Czech)

12. George BM, Nayak SB. Median nerve and brachial artery entrapment in the abnormal brachialis muscle - a case report. Neuroanatomy. 2008;7:41-42.

13. Koshy S, Rabi S, Vettivel S, Indrasingh I. Two anatomical variations associated with potential vascular entrapment in the upper limb. Eur J Anat. 2003;7(2):97-100.

14. Rodríguez-Niedenführ M, Burton GJ, Deu J, Sañudo JR. Development of the arterial pattern in the upper limb of staged human embryos: normal development and anatomic variations. J Anat. 2001; 199(4):407-417. 